\title{
Investigación en colaboración en el campo de la salud mental desde una perspectiva de derechos. Reflexiones sobre método, teoría y enfoque epistémico
}

Collaborative research in the field of mental health from a rights perspective. Reflections on method, theory and epistemic approach

\author{
Ana InÉs Heras \\ (CONICET) Universidad de Buenos Aires \\ Universidad Nacional de San Martín \\ María Cecilia Acosta \\ Universidad Nacional de Rosario \\ maria.ceciliaacosta@hotmail.com.ar (AGENTINA) \\ María Isabel Pozzo \\ (CONICET) \\ Universidad Nacional de Rosario
}

Recibido: 15.062019

Aceptado: 19.11.2020

\section{RESUMEN}

En este trabajo presentamos una discusión de marcos histórico-conceptuales de producción de conocimiento en colaboración que han operado como antecedentes del estudio presentado desde el enfoque de co-investigación. Más precisamente, la revisión de antecedentes avanza en las posturas que abrevan en el binomio saber-poder a los largo de la historia reciente, recuperando los aportes de pensadores/as que han tomado como eje las situaciones de subalternidad político-subjetiva en distintos modos. 
Desde ese marco, focalizamos en investigaciones con sujetos que viven una constante lucha por acceder a sus derechos políticos, sociales y vitales. Luego, damos cuenta del diseño desarrollado en un estudio colaborativo con grupos de trabajo auto-gestionado en salud mental en Argentina. Recuperamos de dicho estudio la sistematización de aprendizajes vinculados a la autogestión en dirección a la constitución micropolítica de la salud mental en tanto derecho ofreciendo una descripción del programa metodológico ordenado en fases de generación de conocimiento en colaboración. Asimismo detallamos luego las potencias surgidas del uso de la co-investigación en salud mental, en tanto posicionamiento conceptual, metodológico y epistemológico en tres vertientes específicas: el ejercicio de la alteridad desde las construcciones y valoraciones en relación al percibido otro, las aportaciones de la etnografía multisituada, y las vinculaciones entre dinámica molecular-molar y enfoque etnográfico para direccionar acciones y decisiones de investigación pero asimismo medidas de salud.

Como cierre, señalamos los aportes sustanciales y distintivos de esta metodología que se aleja de la acumulación de saberes en tanto concibe el conocimiento más bien como posibilidad de transformación de las formas de vida. En ese sentido, situamos en primer término la multi-referencialidad para generar conocimientos y, en segundo término, la capilaridad a la diversidad de narrativas co-creadas, en relación con la democratización de voces y la posibilidad de que el conocimiento generado se coloque en un plano de sentido social-político en referencia al acceso a derechos.

\section{PALABRAS CLAVE}

Etnografía multi-situada; investigación colaborativa; salud mental; autonomía; investigación-acción.

\section{ABSTRACT}

In this work, we present a discussion of historical-conceptual frameworks of collaborative knowledge production that have operated as antecedents of the study presented from the co-research approach. More precisely, the background review advances in the positions that lead to the knowledge-power binomial throughout recent history, recovering the contributions of thinkers who have taken political-subjective subalternity as axes in different ways.

From this framework, we focus on research with subjects who live a constant struggle to access their political, social and vital rights. Then, we give an account of the design developed in a collaborative study with self-managed work groups in mental health in Argentina. We retrieve from this study the systematization of learning related to self-management in the direction of the micropolitical constitution of mental health as a right, offering a description of the methodological program ordered in phases of collaborative knowledge generation. Likewise, we 
then detail the powers arising from the use of co-research in mental health, as conceptual, methodological and epistemological positioning in three specific aspects: the exercise of otherness from the constructions and evaluations in relation to the perceived other, the contributions of the multi-sited ethnography, and the links between molecular-molar dynamics and ethnographic approach to direct research actions and decisions but also health measures.

As a closing, we point out the substantial and distinctive contributions of this methodology that moves away from the accumulation of knowledge as it conceives knowledge rather as a possibility of transformation of life forms. In this sense, we place in first term, the multi-referentiality to generate knowledge; secondly, the capillarity to the diversity of co-created narratives, in terms of democratization of voices and the possibility that the knowledge generated be placed on a plane of social-political meaning in reference to access to rights.

\section{KEY WORDS}

Multi-situated ethnography; collaborative research; mental health; autonomy; action research

\section{INTRODUCCIÓN}

A lo largo de los años 2014-2019, y en el marco de un Programa de Investigación ${ }^{1}$ Colaborativo, desarrollamos en Argentina un estudio en torno a la construcción de la salud mental como derecho humano (Bejarano y otros, 2019). Más precisamente, uno de los estudios que formó parte del Programa, y en el marco de una tesis doctoral, analizó los aprendizajes de y en autogestión en una variedad de dispositivos de salud mental, y las potencialidades instituyentes producidas en dichas realidades institucionales (Acosta, 2019) realizando para ello un proceso etnográfico multi-situado por el cual se procede tanto conceptual como espacialmente a partir de un proceso de de-centración (Falzon, 2009). De este modo, lo que se va produciendo como comprensión se ve informado por una yuxtaposición de análisis e interpretaciones entre sitios y entre resultados parciales, que van permitiendo construir hipótesis más refinadas. Luego de dicho proceso se realizó una estancia focalizada en uno de las organizaciones donde previamente habíamos establecido contacto, para profundizar en aspectos distintivos de ese espacio.

Durante la etapa de etnografías multisituadas nos propusimos interpretar las prácticas, discursos y sentidos llevados adelante en los dispositivos que

${ }^{1}$ Programa de Investigación colaborativa y propiedad colectiva, Aprendizaje de y en autogestión. La autonomía como proyecto humano. Directora: Ana Inés Heras. Subsidiado con fondos PICT0943/2015, fondos del Instituto para la Inclusión Social y el Desarrollo Humano y aportes de organizaciones colectivo solidarias (tiempo, recursos para sostener estancias de campo y transporte). 
identificamos en varias organizaciones, las cuales, a su vez, estaban insertas en contextos institucionales distintos: dos hospitales públicos, una institución privada de atención de la salud mental, dos organizaciones cooperativas y dos organizaciones socio-comunitarias. Los dispositivos habían sido identificados a partir de realizar una serie de entrevistas y análisis de literatura a partir de lo cual construimos un marco metodológico para identificar índices de lo que pensamos junto a Castoriadis (2005) con la denominación de "proyecto de autonomía". En esa perspectiva, y resignificando la propuesta del autor, establecimos que la autonomía como proyecto se distingue por generar espacios y tiempos donde los participantes puedan tener relaciones de paridad, de cuidado mutuo, de participación abierta frente a la creación de las normas que rigen el entramado grupal y de una búsqueda de interpelación de condiciones de dominación.

El diseño de este estudio vinculó las fortalezas propias de la investigación colaborativa con la propuesta de elaborar, junto a los participantes, una metodología para observar el derecho a la salud mental. Dada la vastedad del corpus de información generada, en los límites de este artículo nos interesa señalar algunas consideraciones metodológicas utilizadas en vistas a ponerlas en valor para futuras líneas de indagación en ciencias sociales y humanas. De este modo, focalizamos en tres aspectos: la potencia ética y epistemológica de la co-investigación, el valor instrumental de las etnografías multi-situadas y la articulación entre dinámica molecular-molar y enfoque etnográfico. Para ello, en un primer apartado destacaremos algunos aportes conceptuales y metodológicos de diferentes perspectivas que se han propuesto el desarrollo de investigaciones en colaboración. El interés en desarrollar este primer panorama reside en identificar algunas cuestiones complejas que resultan cuando se trata de investigaciones colaborativas junto a sujetos que desarrollan sus vidas en contextos signados por la constante lucha por acceder a sus derechos políticos, sociales y vitales. En un segundo apartado daremos cuenta de lo que permitió el enfoque etnográfico multisituado en nuestro estudio, desde el punto de vista teórico, metodológico y epistemológico, las construcciones en relación al percibido otro, y las relaciones entre articulación entre dinámica molecular-molar y enfoque etnográfico. Finalizaremos con un breve apartado de discusión y conclusiones en donde nos proponemos por un lado conceptualizar los aportes específicos que el enfoque de generación de conocimiento colaborativo de propiedad colectiva produce en términos conceptuales y metodológicos. Por otro lado, señalaremos los hallazgos más importantes, pero no por ello menos conflictivos, que surgen de un trabajo realizado con diferentes grupos, organizaciones, usuarios y profesionales del sistema de salud mental.

\section{INVESTIGAR JUNTO A OTROS.}

Existe una literatura vasta, en distintas lenguas y producida en geografías diferentes a lo largo del tiempo, que describe, analiza, interpreta y problematiza acerca de las relaciones entre la investigación y la acción. En este apartado 
presentamos algunos puntos centrales de estas orientaciones vinculándolas al trabajo en salud mental.

Escribiendo en castellano y trabajando sobre los desarrollos producidos hasta el momento en Argentina, el trabajo pionero de Grinberg, Langer y Rodrigué en 1957 identificó una serie de perspectivas que, en ese tiempo histórico, estaban convergiendo para desarrollar simultáneamente dos aspectos: el primero, una práctica de salud mental ligada al tratamiento de lo social-histórico según se hacía visible en el tratamiento de grupos terapéuticos, y el segundo, la participación de los directos interesados en una reflexión sistemática sobre lo que la psicoterapia podía ayudar a visibilizar. Estos ejes convergieron a lo largo de la segunda mitad del siglo XX para producir así algunos preceptos ligados a lo que más tarde se identificaría más claramente con investigación acción, investigación participativa y co-investigación. La documentación que realizan esos autores toma como referencia el trabajo desarrollado en Europa, Estados Unidos y América Latina ${ }^{2}$. Se demuestra con ello que en todas esas geografías co-existían inquietudes y desarrollos parecidos.

En Argentina estos primeros posicionamientos dieron lugar más adelante al rompimiento de varios profesionales con la Asociación Psicoanalítica Argentina, justamente por cuestiones vinculadas a los modos del poder autoritario y su vinculación con el saber (Rodrigué, 2000). En forma paralela y algo diferente, los desarrollos de Pichón Rivière (1969 a, 1969 b, 1971, 1995, 2008) en la fundación y fundamentación de su programa de trabajo ligado a la psicología social también pusieron de relieve que el aprendizaje desarrollado en grupo — bajo ciertas condiciones - permite una co-investigación sobre cualquier contenido (que Bleger y Rivière denominaron aprendizaje) y una interrogación, simultáneamente, de los modos en que esa co-investigación se desarrolla. Quiroga (1986) ha detallado cómo, ya en el principio de esas fundamentaciones desde fines de los años 50, Rivière trabajaba con la hipótesis de que el proceso de aprendizaje permite sostener la buena salud (física, mental y emocional), y que para que dicho proceso se verifique, es necesario reconocer la naturaleza social y colectiva de la construcción de conocimiento, tornando explícitas las condiciones en que se produce. Varios de los preceptos que Rivière desarrolló más adelante fueron probados primero en grupos de trabajo en psiquiatría y medicina que él mismo coordinó, donde se modificaron, adrede, los modos de interacción entre Profesor-médico y alumnos, para producir relaciones horizontales en función de generar conocimiento en forma conjunta (Fabris, 2007, 2009 y 2014).

Los movimientos simultáneos en campos disciplinares complementarios pero algo diferentes tales como la psicología social, el análisis institucional, la psicoterapia de grupo y el psicoanálisis ligado al marxismo, fueron conceptualizados en ese momento como ligados al freudo marxismo por el filósofo argen-

2 Deberíamos agregar a estas locaciones la del norte de África, con el trabajo realizado por Fanon, quien fue discípulo de Francesc Tosquelles y contribuyó a desarrollar el planteo teórico en relación al poder violento racista y la enfermedad mental, es decir que auscultó el ángulo del poder social y político y puso en visibilidad los efectos de esa dominación. 
tino León Rozitchner (2012 [1972]). Esta producción conceptual se producía en décadas de cambios veloces (fines de los años 50 y mediados de los 70), cuando en muchos países de América Latina, el avance de la izquierda política y cultural hizo posible algunas experiencias nacionales fuertemente informadas por un cuestionamiento a las jerarquías, en distintos campos: el arte y la cultura, la salud, la salud mental, la educación y la producción científico técnica. Por ejemplo, es en esos años que Varsavsky escribe Ciencia, política y cientificismo y aboga por una generación de conocimiento que permita independizar la mente y las acciones de toda dominación. También Orlando Fals Borda, en esas mismas décadas del siglo pasado, realizó una labor muy importante de, por un lado, proponer y realizar estudios con enfoque participativo, y por otro, de coordinar y dirigir una serie de procesos institucionales en la universidad en Colombia, además de su participación en la política de su país. Algunos de los aspectos más relevantes con respecto a su conceptualización y acción en investigación participativa han sido sintetizados en Fals Borda (1999).

En tanto en Chile, Argentina, Uruguay, y también Brasil, tuvieron lugar fenómenos de extrema violencia asociados a las dictaduras cívico-militares que asesinaron a miles de personas y empujaron a otros cientos de miles al exilio. Estos procesos sufrieron la paradójica doble situación de haber sido intervenidos y truncados en algunos países, y revitalizados en otros, precisamente por efecto de los exilios. De esta manera, por ejemplo, México y Brasil son tributarios del exilio chileno, argentino y uruguayo durante los años 70 , y en esos espacios se reconfiguraron construcciones de acción y pensamiento que habían sido originadas, por ejemplo, en Argentina. Baremblitt documenta parte de esto en un trabajo que escribe más tarde, en 1982, y concluye que de estas líneas se desprenden en Argentina, Uruguay y Brasil vertientes que ligan la práctica clínica terapéutica, el arte, el pensamiento y la investigación con y para los grupos, y que a la vez toman muy seriamente tanto la producción de conocimiento como el rol del psicoanalista, médico, investigador y enfermero como trabajadores de la salud pública, un aspecto distintivo de estos países. Una de esas líneas es el movimiento institucionalista, y dentro de él o relacionado a él, distintas corrientes de pensamiento y acción: esquizoanálisis, análisis institucional, socioanálisis, entre otros que luego volvieron a recuperarse en Argentina a partir del año 1984 (Saidón, 2012; 2015).

Otros antecedentes ligados tanto al campo psi- como al educativo, son las propuestas de Paulo Freire (1969), María Teresa Sirvent (1990) e Ignacio Martín Baró (1986). Si bien trabajan en distintas disciplinas y países, y además migran en distintos momentos de sus vidas, tienen planteos en común en relación a la relación saber-poder, la potencia emancipadora del conocimiento y la perspectiva crítica. En estas líneas es preciso mencionar también el trabajo de Enrique Dussel, uno de los ejemplos que mencionábamos antes, ya que debe migrar de Argentina a México. Produce desde la filosofía, informada por la teología de la liberación (1973), una epistemología propia de América Latina vinculada a lo que Rodolfo Kusch (2000) llamó la América Profunda.Esta refiere a modos del 
saber, del conocer y del hacer que se asientan en formas mixtas e informadas fuertemente por perspectivas ancestrales.

Con posterioridad, y algo diferente en el planteo pero abrevando también en el binomio saber-poder, saber ancestral, conocimiento etno-local y emancipación, en América Latina se desarrolla un tipo de trabajador/a de la investigación ligado a clases populares, pueblos indígenas y grupos tratados como "menores". Ejemplo de esto son mujeres y jóvenes cuyos derechos han sido vulnerados, personas con una identidad de género que no es "unívoca", etc.. De estas corrientes podemos destacar el trabajo de tres mujeres: Raquel Gutiérrez Aguilar, Rita Segato y Silvia Rivera Cusicanqui, quienes desde los años ochenta hasta la actualidad, produjeron una investigación con y sobre diferentes sujetos subalternizados, promoviendo a la vez la comunicación de saberes singulares, y la co-investigación, tomando además en cuenta que el conocimiento generado sea útil al cambio social.

En el contexto de habla inglesa fue Kurt Lewin (1946, 1947a y 1947b) quien introdujo la noción de acción ligada a la investigación, a su vez inscripta en el cambio social. Lewin falleció inmediatamente al año siguiente y por lo tanto, el pensamiento y elaboración sobre este enfoque no fueron debidamente sistematizados por el mismo autor, aunque hubiera usado antes otros términos con un sentido conceptual similar: investigación cooperativa e investigación en acción. De tal modo, es legítimo considerar a Lewin uno de los precursores en este tipo de enfoque en el campo de la investigación ligada al trabajo en y con grupos, al alivio del malestar y a la transformación político social en el mundo angloparlante. Bargal (2006) sostiene que el desarrollo de este marco conceptual y metodológico en Lewin estuvo informado tanto por su posición existencial en el mundo como por su formación en campos disciplinares combinados. La vida de Lewin estuvo atravesada por la discriminación por su condición de judío y luego esto implicó su desarraigo y migración a los EEUU. Durante su trabajo en este segundo país, tuvo la oportunidad de continuar desarrollando un marco que ya era inter-disciplinar originalmente, en un cruce entre psicología, filosofía, antropología y sociología. Sin embargo, no fue sino bastante más tarde que articuló estas relaciones disciplinares para fundamentar su programa de trabajo en el Massachusetts Institute of Technology donde trabajó entre 1944-1947. Su preocupación fundamental en esos años eran las relaciones inter-grupales, en particular, situaciones donde existieran cuestiones de discriminación de un grupo hacia otro. Es en este contexto que se interesó por profundizar y comenzar a sistematizar su metodología colaborativa, inclusive nombrándola como action-research, el modo en que sería más adelante mundialmente conocida. Sus principios fundamentales tienen que ver con la colaboración para la generación de conocimiento, la relación entre momentos de trabajo intelectual y momentos de trabajo que implican la puesta en juego del ser en sentido holístico, la interrogación constante sobre el contexto singular donde se está produciendo el conocimiento a la vez que se mantiene la pregunta sobre leyes generales sociales (esto último inspirado en el marco epistemológico de Lewin que vincula la empiria al desarrollo de abstracciones y leyes generales de desarrollo social). 
A partir de este desarrollo, durante los años 60 y 70 en los Estados Unidos, se conceptualizaron estos primeros acercamientos a la metodología y se diversificaron tanto los problemas estudiados (es decir, más allá de las relaciones inter-grupales) como los campos disciplinares que contribuyeron a forjar las teorías y metodologías de trabajo y las formas de nombrar los enfoques que surgían así de estas prácticas. Uno de los enfoques más comúnmente referidos es del de la investigación-acción participativa o PAR por sus siglas en inglés. Según Genat (2009), en esta forma de trabajo se plantean tres cuestiones fundantes: lo que se investiga es la acción investigativa (action-research) de participantes que colaboran entre sí para generar conocimiento en un contexto singular. Para ello se trabaja en ciclos de acción y reflexión que producen un aprendizaje basado en la experiencia de forma tal que su orientación es la de generar un proyecto emancipatorio. Por último, lo que surge como producción es no solamente una investigación concreta y localmente situada sino un marco conceptual que permite continuar el proceso.

Estos antecedentes conceptuales permiten situar el trabajo que se realizó en la ciudad de Rosario, Argentina. Hubo una etapa previa de exploración (20112013) y otra de estudio propiamente dicho, durante 2014-2019. Es necesario acercar una nota sobre la relación entre los modos de hacer investigación desde sus vertientes democratizantes y colaborativas que venimos detallando aquí y el enfoque de Derechos Humanos en salud mental planteado en Argentina, cuyo instrumento de política pública es la Ley 26.657. Hemos partido de la perspectiva de que la salud mental es un derecho de toda persona y no un bien de consumo o mercancía. Dicha concepción es heredera de los movimientos y reformas que a lo largo de la historia latinoamericana reciente produjeron las legitimaciones de una salud mental concebida en un enfoque de derechos, y se inscribe también en otros instrumentos internacionales, tales como la Declaración de Caracas de la Organización Panamericana de la Salud (OPS, 1990), los Principios de Brasilia Rectores para el Desarrollo de la Atención en Salud Mental en las Américas (OPS, 2005), la Estrategia y Plan de Acción de Salud Mental (OPS, 2009) y el Consenso de Panamá (OPS, 2010).

En el contexto de estas elaboraciones tendientes a democratizar las prácticas de salud mental con una perspectiva de derechos, la Ley 26.657 detalla las acciones de inclusión social, laboral y de atención en salud mental comunitaria, desde la articulación de los sectores de salud, trabajo, educación, desarrollo social, en cada jurisdicción de la nación argentina. Esta Ley ha permitido disponer de una noción de salud mental congruente con la complejidad que subyace al recorte de estudio cuyos detalles se plantean en este artículo a nivel conceptual y metodológico, en la medida en que se comprende a la salud como una articulación entre una multiplicidad de componentes de naturalezas diferentes (biológica, psicológica, socio-cultural y económica). Se debe propender asimismo al pleno goce de los derechos civiles y sociales del usuario de salud mental. Con ello marca una orientación inclusiva y apunta a la sustitución progresiva de los hospitales monovalentes por dispositivos alternativos al manicomio a través de la concreción de acciones de inclusión, dando sustento a los procesos de desmanicomialización. 
Estas acciones de inclusión dan sustento a la concreción de derechos que supone la salud mental pensada en la complejidad de sus componentes. Se favorece la autonomía de los usuarios de salud mental en tanto se limitan las internaciones, en base a la presunción de capacidad de los usuarios habilitante para ejercer y/o restituir su estatuto de ciudadanía y esto supone también el pleno ejercicio de derechos sociales tales como derecho a la salud, a la vivienda, a la ciudad, que se conciben vinculados entre sí. Le corresponde al Estado su garantía integral, según la legislación.

Se detalla en las secciones siguientes la importancia de comprender la cualidad específica de los dispositivos de integración, que dan consistencia al derecho a la salud mental aptos para desarrollar un trabajo en toda su complejidad. En este sentido, a continuación se presentan —en su intervinculación- las cuestiones asociadas al ejercicio de la alteridad desde las construcciones y valoraciones en relación al percibido otro, las aportaciones de la etnografía multi-situada y las vinculaciones entre dinámica molecular-molar y enfoque etnográfico.

\section{CO-INVESTIGAR EN SALUD MENTAL}

Durante 2011-2019 se llevó adelante un proceso de investigación que se diseñó originalmente en tres etapas vinculadas entre sí. En la primera etapa exploratoria (2011-2014) se desarrollaron algunas actividades (entrevistas, observaciones, lectura de material bibliográfico y contactos con profesionales y organizaciones de salud mental) para ir construyendo preguntas a abordar en etapas posteriores en conjunto con distintos participantes. La idea central desde el inicio era poder indagar en las relaciones entre autogestión y derechos humanos en salud mental. Se había partido de suponer que existía una experiencia vasta en Rosario, provincia de Santa Fe, que permitiría informar tanto a los usuarios del sistema (beneficiarios, sus familias, profesionales y otros actores involucrados) como a quienes hemos venido trabajando junto a una variedad de grupos y organizaciones que luchan constantemente por el ejercicio pleno de derechos en torno a salud, vivienda, alimentación, trabajo, educación, arte y recreación.

A partir de esa primera etapa de colaboración y exploración, durante 20142017 se trabajó junto a diferentes grupos y profesionales profundizando en las preguntas que luego se abordaron en mayor profundidad durante 2017-2019. Dichas preguntas giraron en torno a los aportes de la auto-organización al alivio del malestar psíquico, al lugar del arte y la cultura en dichos procesos, y al acceso a derechos en relación al alivio del malestar. En estas etapas, el diseño metodológico, conceptual y epistémico se fue construyendo en colaboración con distintos participantes, y cada proceso de relación con cada grupo, o con cada profesional, se fue desarrollando en el mismo quehacer. De este modo, esta metodología tiene en cuenta al mismo tiempo la singularidad de cada caso (grupo, organización, contexto) y una premisa general: que en cada situación se produce conocimiento situado que sin embargo permite informar otras situaciones o problematizar cuestiones que no pueden verse en algunos contextos inmediatamente, pero que 
aparecen señaladas por contraste. Esta metodología se vincula con el modo de trabajar que tuvo Dell Hymes durante los años 80 en Pennsylvania, quien desarrolló un enfoque denominado colaborativo, contrastante y en sumatoria de casuística (nuestra traducción, de su expresión original collaborative / contrasting / accumulative). Esta perspectiva es congruente con algunas propuestas de etnografías educativas en América Latina a partir de esa misma década.Algunos de estos enfoques se orientaron además algo más tarde a pensar las relaciones internas en los equipos de investigación y también pensar en profundidad quiénes están generalmente habilitados para realizar investigación y quiénes no, cuestionando esta división de roles en un quehacer colaborativo de propiedad colectiva (ver Heras, 1993, 1995, 1999 y 2014).

A continuación damos cuenta, descriptivamente, del trabajo realizado en las etapas de nuestro trabajo, planteando en cada una qué tensiones, problematizaciones y preguntas se fueron haciendo visibles, y cómo éstas permitieron continuar forjando el modo de trabajo con los distintos participantes a lo largo del tiempo. Estas preguntas y tensiones también permitieron examinar algunos presupuestos teóricos y metodológicos, tales como la vinculación (que vimos estrecha) entre un enfoque etnográfico colaborativo para documentar procesos cotidianos y los postulados teóricos de la filosofía Deleuzo Guattariana en su énfasis en considerar la relación entre lo molecular y lo molar. A lo largo del texto iremos presentando algunos aspectos que harán visibles estas relaciones teóricas entre etnografía, colaboración y filosofía.

Entre 2011 y 2012 nos pusimos en contacto con varios/as profesionales y usuarios del sistema público y privado de salud mental, tanto en Rosario como en Ciudad Autónoma de Buenos Aires (CABA), Córdoba y Entre Ríos. En estas primeras conversaciones exploratorias surgió un aspecto interesante y difícil de abordar con respecto a las relaciones entre usuarios directos (beneficiarios y sus familias), organizaciones que luchan por sus derechos, y profesionales que participan tanto del sistema como de la lucha. El aspecto clave de esta etapa lo constituyó lo que en ese momento denominamos la voz y la toma de la palabra / su distribución. En esos momentos, es necesario recordar, la Argentina atravesaba la etapa de la reciente sanción de la Ley de Salud Mental (2010) y se habían establecido diferentes modalidades para trabajar los alcances de la Ley. Entre ellas, existían convocatorias a Asambleas. Una de las autoras de este trabajo participó de estos espacios bajo la premisa metodológica de la participación directa en la realidad de estudio. El tránsito por estas instancias asamblearias nos mostró el acceso a grados de auto-organización por parte del colectivo de usuarios/as de salud mental.

La evidencia generada dio cuenta del proceso de empoderamiento de los usuarios/as en términos de ejercicio de la autonomía y producción de la salud mental como derecho, modos de conceptualizar que surgen de un análisis con categorías mixtas, es decir, con términos usados y definidos por los participantes directos originalmente auto-convocados y por una de las autoras, en tanto también participante de esa experiencia. Este hallazgo resultó significativo para orientar epistémica y conceptualmente nuestro trabajo y también para dialogar 
con los usuarios del sistema de salud mental y sus familias. Asimismo, produjo tensiones hacia el interior del espacio ya que se pusieron de manifiesto, a través de estas conceptualizaciones, distintas versiones de lo que significaba en ese momento y lugar la auto-organización. Más precisamente permitió comprender el proceso de lucha de los usuarios/as en tanto salida de una posición de subalternidad y resistencia ante el avance hegemónico del paradigma biomédico psiquiátrico. Para ello, pusimos en valor las narrativas de los participantes del colectivo de usuarios/as y optamos por incluir en nuestro marco teórico los aportes de la literatura elaborada por autores que se referencian como usuarios/as o que a lo largo de la historia reciente generaron una obra literaria en situación de encierro manicomial o medicalización.

Asimismo, a la hora de abordar analíticamente un vasto corpus de evidencia generada en colaboración, pudimos considerar la pertinencia de leer el material utilizando el par conceptual "distribución del poder-saber y empoderamiento del usuario". Esta categoría formó parte de la matriz analítica y fue creada a partir de identificar el avance autogestionado de los usuarios/as en la defensa de sus derechos, conceptualizados por ellos mismos como derechos humanos, y su afirmación identitaria en tanto colectivo de lucha. El reconocimiento de las construcciones de procesos de subjetivación (Tassin, 2012) de los usuarios/as a través de la ponderación de su discurso, su voz y posición de trabajo en horizontalidad hacia la autonomía, alcanzó un sentido democratizante en la circulación de poder y saber propia del ámbito de la investigación en salud mental, por lo que podemos interpretar estos procesos, junto al autor citado más arriba, como procesos de subjetivación política. Este aspecto permite, nuevamente, establecer vínculos entre la potencia de la etnografía (en su capacidad de hacer visible lo que parece natural o de sentido común) y la filosofía política Deleuzo Guattariana (en su insistencia en ligar procesos que tienen lugar constantemente y son por ello más bien indeterminados, con procesos que tienden a estructurarse molarmente y por ello tienden a una cierta inmovilización social). En esta etapa pudimos identificar aspectos que constituían acciones de reclamo y de lucha por garantía de derechos por parte de usuarios, familias y algunos trabajadores de la salud mental: solicitud de acceso a propuestas culturales, laborales y educativas; solicitud de que existan estructuras públicas más amigables con respecto a la inclusión sociolaboral; cuestionamiento del uso de la jerga médica para dirigirse a personas cuya comprensión de dicho modo de hablar no es inmediatamente posible.

En una etapa posterior de la investigación desarrollamos un trabajo multi-situado, de carácter etnográfico, junto a una variedad de organizaciones. Partimos de sistematizar los espacios con quienes se pudiera realizar un trabajo conjunto en base a las preguntas de investigación. Desde el momento en que comenzamos con este relevamiento, del cual participamos dos de las autoras de este trabajo, decidimos operar desde la mencionada posición de multi-situación, por dos motivos. El primero, porque hipotetizamos que en esa suerte de dis-locación encontraríamos riqueza interpretativa puesto que los sitios donde proponíamos trabajar tenían diferentes definiciones, inclusive vocabulario, para referirse a quiénes participaban, cómo lo hacían y qué hacían en conjunto. De este modo, el 
primero punto era de orden epistémico. El segundo fue que presuponíamos que el vínculo que iríamos forjando en cada caso permitiría más o menos posibilidad de trabajo conjunto, colaborativo y de participación directa, con lo cual este otro aspecto fue de orden metodológico.

Fuimos identificando distintos espacios de trabajo (clínicas privadas, hospitales públicos, organizaciones sociocomunitarias, organizaciones de usuarios y cooperativas de trabajo en salud mental) y a su vez, en ellos, tomamos la perspectiva de describir y comenzar a analizar los dispositivos que se alojaban, buscando comprender las transversalidades y distinciones entre ellos. Produjimos durante esta etapa de trabajo un corpus de conocimiento en colaboración: observaciones, entrevistas individuales y colectivas, participaciones directas en actividades grupales que tomaban el propósito de la auto-organización. Por ejemplo, durante el año 2015-2016 participamos de las reuniones de colectivos de defensa de derechos de usuarios/as de salud mental, encuentros nacionales y jornadas comunitarias fértiles para avanzar de manera coherente con nuestro interrogante en torno a la construcción de la salud mental como derecho. También realizamos observaciones y trabajo en las distintas organizaciones mencionadas y en cada caso tuvimos que tomar decisiones conceptuales y metodológicas de qué significaba la colaboración. De esta manera, y aquí surge un punto de orden teórico, la investigación colaborativa, si bien tiene ciertos presupuestos conceptuales, no puede nunca terminar de definirse sino hasta que ocurre. De este modo, sus capacidades de generar conocimiento ocurren en el durante y su práctica se aprende haciéndola (Pozzo, Miano, Heras, 2018).

Durante esta fase, el rastreo de los aprendizajes de autogestión puso en evidencia una gran diversidad de modos que tenían lugar en lo cotidiano. Junto a los colectivos de trabajo, fuimos encontrando que existían posiciones heterogéneas con respecto a realización de la autogestión y el ejercicio de la autonomía. Para abarcar esta diversificación construimos una tipificación que será detallada más adelante.

Señalamos una tercera etapa de investigación que denominamos estancia etnográfica propiamente dicha. En este momento se decidió profundizar el trabajo en una de las organizaciones sistematizadas para alojar una estancia etnográfica más a fondo, por las razones epistemológicas, metodológicas y teóricas explicadas más arriba. Es decir, en este caso, este lugar permitía profundizar en las cuestiones que se habían ido analizando y sobre las cuales se habían ido produciendo transformaciones en nuestro marco de trabajo y metodología. Nos orientamos por los resultados del análisis anterior que evidenciaron la relevancia del acceso a la palabra, la creación artística y la co-construcción de una potencia vinculada a la salud mental. En base a la consideración de estos aspectos, fuimos analizando más a fondo la potencia de la creación artística en el marco de la construcción de salud mental hacia la autonomía en el sitio elegido y co-construido como lugar de reflexión por parte de nuestro equipo, el equipo de trabajo del hospital público donde se alojó la experiencia, y los usuarios que concurrían.

La estancia etnográfica propiamente dicha nos aportó indicios de acciones de transformación de situaciones dadas, instituyentes, que interpretamos en base 
a tres ejes: clínico, ético y político. Con estos tres elementos construimos un patchwork metodológico al modo de un instrumento analítico apto para abarcar la complejidad de los datos reflejada en esas tres dimensiones. Optamos por esta perspectiva porque a esta altura de la investigación, se había hecho visible que es necesario operar metodológicamente a través de distintas herramientas, sin desconocer el origen de cada una de ellas, y a la vez sin aplanar o someter ninguna de ellas a alguna de la otra. Por ejemplo, el hecho de haber realizado las etnografías multisituadas, como dijimos, nos había permitido comprender que los términos localmente utilizados en cada una de las experiencias, y las prácticas o situaciones que describían, era de orden situado contextualmente, y resignificado en la relación entre usuarios, profesionales, otros profesionales de la organización (ejemplo, hospital público), familiares y equipo de investigación. De este modo, nuestro trabajo etnográfico en profundidad, si bien partió de tomar esa información de las etapas anteriores, también buscó profundizar en cuestiones problemáticas de ese sitio, tales como la relación salud mentalhospital público, intersubjetividad-relación "tradicional" médico paciente, o salud pública-derechos humanos. Nos propusimos trabajar así a partir de estos tres interrogantes: ¿Qué sustentos encontramos para pensar una clínica al servicio de la construcción de salud mental como derecho? ¿Qué aspectos éticos del tratamiento del otro en la intersubjetividad construyen salud mental como derecho? Y, la inscripción política de un dispositivo en el proyecto de autonomía ¿aporta a construir salud mental como derecho y cómo en caso de que sí?

La perspectiva de la co-investigación orientó nuestro diseño de trabajo en términos epistemológicos y éticos. Generar conocimiento en colaboración con otros/as fue un postulado útil para plantear la construcción de saberes desde la intersubjetividad, en los encuentros con interlocutores distintos. Estos saberes constituyen la evidencia en torno a cómo diversos aprendizajes orientados por la autogestión pueden pensarse como constructores de salud mental. Pero además incluyen la posibilidad de investigar con las diferencias de repertorios subjetivos propias de los compañeros de estudio. La riqueza de este modelo nos permitió tender los vínculos en horizontalidad. Esta mirada nos ha permitido soslayar la premisa de recolección de datos propia de enfoques de investigación más tradicionales, facilitando la posibilidad de pensar la construcción del campo de estudio como emergente de la dinámica propia de las interacciones realizadas en entrevistas, observaciones y participaciones directas en los dispositivos de salud mental. Allí radica su potencia epistemológica. Por otra parte, la adopción de esta perspectiva de trabajo permite la circulación del poder y el saber en horizontalidad en tanto supone al otro como interlocutor con la capacidad de agenciar la producción de saber. Aquí el conocimiento es una producción que emerge entre subjetividades que no mantienen una jerarquía.

Como resultado del estudio expuesto, hemos avanzado en la identificación de situaciones que nos permiten sostener una vinculación entre el proyecto de autonomía, la política pública y la salud mental. Esta vinculación posibilita corroborar que existen formas específicas a través de las cuales la autonomía como 
proyecto vital colabora a construir una lógica de inclusión social plena, condición que a su vez habilita el ejercicio de la salud mental como derecho.

En este sentido encontramos que el proyecto de autonomía se corrobora en situaciones que convocan a conmover lo instituido, es decir, instancias de cuestionamiento ante lo dado precisadas en la modificación de las condiciones de encierro manicomial por parte de los trabajadores de salud mental. El material de entrevistas y observaciones arroja evidencia a este respecto, por ejemplo, en la documentación de narrativas que refieren acciones de resistencia y creación a través del desarrollo de acceso a bienes culturales. Consideramos la potencia testimonial de estas narrativas en tanto hacen consistir los derechos civiles y sociales (en este caso, cultura y educación, en interdependencia con salud), y también su valor para proveer evidencia en lo que respecta a nuestra interpretación sobre los aprendizajes de autogestión (cultural, social, afectiva) producidos en instancias de intersubjetividad que sostienen el armado de vínculos solidarios en condiciones de horizontalidad. Con ello hemos podido verificar que el tendido de vínculos en horizontalidad (rasgo central de la autonomía cuando se la observa en proyectos colectivos) se presenta como facilitador para concretar acciones de salud mental en tanto derecho.

Estamos ahora en condiciones de detallar lo que permitió el enfoque de nuestro estudio, desde el punto de vista teórico, metodológico y epistemológico, en relación a tres aspectos: las construcciones en relación al percibido otro, las aportaciones de la etnografía multisituada y las relaciones entre articulación entre dinámica molecular-molar y enfoque etnográfico.

\subsection{Construcción de conocimiento en relación al percibido otro.}

La búsqueda de la horizontalidad entre sujetos de investigación es un rasgo propio del enfoque colaborativo (Heras y Acosta, 2018). La consideración del otro como semejante supone además la posibilidad de articular un uso metodológico del proyecto de autonomía en tanto este aporta el sentido de democratizar y proponer la igualdad en el acceso a las construcciones semánticas desde la participación igualitaria. Plantear un ordenamiento intersubjetivo desde la horizontalidad y la paridad nos permitió desarrollar un tratamiento del otro respetuoso de las diferencias, que ha dado lugar a la singularidad de cada momento de investigación. Por eso señalamos una potencia ética como parte de las riquezas de la co-investigación. Con este marco, la otredad aparece visibilizada en distintos momentos. Un ejemplo de ello constituyen las decisiones metodológicas asumidas para dar respuesta a uno de nuestros objetivos de investigación destinado a generar conocimiento útil para diferentes actores. En ese sentido, a la hora de elaborar las entrevistas a realizar consideramos la heterogeneidad de voces. Así, el corpus de evidencia generado junto a especialistas se enriqueció con la información que nos aportaron los encuentros con los llamados referentes, a saber, personas que no necesariamente están vinculados al mundo académico ni poseen saber especializado o profesional, cuyas voces han sido puestas en valor 
en base a su implicación en la realidad de estudio. Como resultado de la realización de estas entrevistas alcanzamos la inclusión de los saberes-otro, diversos a los instituidos y hegemónicos. Algunos ejemplos concretos aparecen en los momentos de trabajo en colaboración con trabajadores que asumen la coordinación de dispositivos culturales. Estos espacios construyen salud mental desde ámbitos más amplios que el estrictamente psicológico o médico. Junto a ellos fuimos constituyendo evidencia de cómo una variedad de espacios socio-culturales tales como, dispositivos de intercambios y encuentros dedicados a compartir un momento agradable en disposición de ronda, talleres de desarrollo de la libertad de expresión en distintas vertientes, conformación de instancias colectivas de discusión bajo la modalidad asamblearia, por mencionar algunos, van realizando un armado posible en el acceso a la salud mental como derecho. Dicho acceso se produce bajo las distintas modalidades que ofrece la autogestión en tanto "aprendizaje cultural continuo, asociado a la creación” (Heras, 2014: p. 151).

La inclusión de lo que denominamos saberes-otro, en tanto conocimiento no hegemónico, se precisa además de una manera más clara en la ponderación de las narrativas de usuarios/as del sistema de salud mental a lo largo de la fase etnográfica. En base a los enunciados de los participantes del taller con el que se trabajó realizamos un análisis en profundidad que permitió la identificación de un enfoque en salud mental vinculado al arte que no sigue la lógica de la patologización y segregación de las personas con padecimiento subjetivo.

\subsection{Aportes de la etnografía multisituada como dislocación espacial y conceptual.}

La instancia de generación de datos en terreno fue estructurada en tres momentos sucesivos: fase piloto, trabajo multi-situado con organizaciones, y estancia etnográfica propiamente dicha, como ya mencionamos. Ya también antes indicamos el valor del uso instrumental de las etnografías multi-situadas en la segunda fase de generación de datos, realizada en dispositivos diferentes, que abarcó asimismo la sistematización de organizaciones con quienes se trabajó en conjunto en base a las preguntas de investigación. Fue también en esa instancia que identificamos espacios aptos para realizar observación participante y entrevistas en tres modalidades: con especialistas, con referentes y colectivas, y grupos de participación conjunta con usuario/as y profesionales de salud mental. Optamos por la multi-referencialidad para desarrollar trabajo etnográfico en base al relevamiento cruzado entre piezas de normativa atinentes a la política pública nacional de salud mental y las narrativas producidas junto a referentes y especialistas en la materia. A partir de dicho relevamiento surgieron referencias a dispositivos tales como colectivos de defensa de Derechos de los usuarios/as e salud mental, rondas de discusión con disposición asamblearia, jornadas de trabajo comunitario en salud y encuentros nacionales de usuarios/as y trabajadores. La etnografía multi-situada nos permitió trabajar en colaboración con todos ellos. 
Este enfoque también permitió, como se anticipó más arriba, establecer una combinación de acercamientos con las organizaciones. Ejemplos de esto son: las observaciones participantes junto a un colectivo de estudiantes y usuarios/as que desarrolla una propuesta de proyección de cine en el interior de un hospital monovalente; las entrevistas individuales con especialistas en salud mental tales como psicoanalistas, esquizoanalistas, analistas institucionales, musicoterapeutas y psiquiatras y las entrevistas colectivas con organizaciones que proponen un trabajo de salud vinculado a la creación literaria y a la expresión corporal. Asimismo, conversaciones por otros medios (correo electrónico, telefónico y whatsapp) con integrantes de dispositivos culturales dedicados a salud mental. Estos modos diversos se fueron llevando a cabo en forma escalonada respetando los modos en que cada organización pudo ir alojando la tarea en colaboración diversificada en las formas mencionadas.

Durante este tramo del trabajo realizamos además un mapeo global de alcance amplio para sistematizar las organizaciones de salud mental orientadas por la autogestión. Este corpus de información nos ofreció un marco para contextualizar una pieza más específica, presentada como aporte instrumental de nuestra investigación: la tipificación de experiencias de autogestión. El trabajo multi-situado expuso una diversidad significativa en términos de modalidades diferentes de pensar y hacer una práctica de salud informada por la autogestión. Por lo tanto, con la premisa de sistematizar este aspecto elaboramos una tipificación de institucionalidades bajo la cual fuimos inscribiendo los modos diferenciales de alojarse en la autogestión de los colectivos con los que se trabajó. La construcción de esta tipificación es uno de los resultados de la etnografía multi-situada, ya que facilitó la categorización de organizaciones integradas por actores sociales variados de acuerdo al tipo de institucionalidad; es decir, es una construcción que surge de la co-investigación.

De este modo podemos afirmar que la figura de la auto-gestión, en experiencias de salud mental, contiene cuatro modalidades:

A. Espacios auto-gestionados, como cooperativas de trabajo u organizaciones de la sociedad civil dedicados al alivio de padecimientos para con distintos usuarios que acceden a esos servicios.

B. Espacios auto-organizados dentro de instituciones jerárquico-burocráticas (públicas y privadas), llevados adelante o bien por los profesionales que coordinan distintos servicios de salud en esos espacios, o bien por los profesionales en conjunto con los usuarios, o con los usuarios y sus familias.

C. Espacios auto-organizados en torno a temas relacionados a la Ley de Salud Mental y los derechos humanos.

D. Espacios transversales, que presentan una combinación de rasgos específicos de los tres tipos de institucionalidad dando lugar a otra categoría dentro de la tipificación propuesta. 


\subsection{Dinámica molecular-molar y etnografía(s).}

Un tercer aspecto que nos interesa exponer aquí es la articulación entre la dinámica molecular-molar y el enfoque etnográfico en tanto fortaleza del posicionamiento de la co-investigación. En tanto aportación original de nuestro trabajo, este cruce involucra elementos conceptuales y metodológicos útiles para abarcar analíticamente la complejidad de los datos generados en las instancias diversas de la co-investigación. Más precisamente, seguimos esta orientación para construir una serie analítica que pudiera constituir un instrumento apto para leer el alcance de las realidades instituyentes dadas en una escala cotidiana y micropolítica y poder pensarlas como productoras de efectos a nivel de escalas de sentido más amplias, macropolíticas. En ese sentido nos preguntamos por la cualidad específica de los datos generados en colaboración a la hora de conmover las grandes estructuras, tales como la lógica manicomial y heterónoma con la cual entraban en tensión bajo el signo de la autogestión para construir medidas de salud mental. Es decir, nuestros datos testimonian cómo las medidas micropolíticas se presentan como salidas para efectuar acciones de salud mental autogestionadas en un alcance pequeño, molecular, que sin embargo permiten instituir a través de esas acciones contextualmente situadas una otra política concreta orientada por la autonomía. Fundamentamos esta propuesta desde la lógica molecular-molar (Deleuze y Guattari, 1988) operando en combinación con el enfoque etnográfico, que nos permite abordar las grandes preguntas sociales a través de mundos pequeños (Rockwell, 2005).

Desde el linaje conceptual deleuzo-guattariano, los movimientos moleculares modifican los segmentos de las grandes estructuras de sentido y praxis. En ese sentido, Guattari y Rolnik (2005) abrevan en la cuestión micropolítica reconociendo una doble condición para las luchas sociales, lo molar y molecular en tanto planos de constitución en íntima intervinculación. En el marco del estudio aquí expuesto, por un lado, este interjuego se actualiza en las acciones planteadas en la trama de la vida institucional, medidas que construyen autonomía desde las escenas cotidianas y que contienen en sí mismas en base a la dinámica molecular-molar la potencia de expandirse y conmover la lógica más amplia (manicomial en muchos casos). En un orden epistémico y metodológico, el par semántico molecular-molar construyó un apoyo para valorar las acciones de coinvestigación en su posibilidad de transformar la realidad de estudio desde las actuaciones de investigación (pequeñas y plasmadas en la vida cotidiana) propias del enfoque etnográfico y colaborativo.

A su vez este aspecto nos permite poner en vinculación la investigación colaborativa con el acceso a los derechos humanos. Más precisamente, en base a los datos generados pudimos concluir que la construcción del derecho a la salud mental se manifiesta en una escala micropolítica, a partir de acciones pequeñas producidas en los colectivos de trabajo. Estas medidas, que entendemos como autogestionadas, construyen una política pública de salud mental que se proyecta hacia la autonomía. En nuestro corpus de evidencia encontramos ejemplos de estas medidas que tienen la característica en hacer consistir la micropolítica 
como salida ante escenarios de encierro. Dentro de las entrevistas reconocemos el armado y gestión de salidas colectivas del hospital monovalente. Entre las observaciones realizadas ubicamos además el montaje de un dispositivo de cine y debate dentro del escenario manicomial. Otros materiales de entrevistas informan respecto de movimientos pequeños de empoderamiento a través de la toma de decisiones y el reposicionamiento de los "pacientes" que comienzan a asumir un rol más activo en el tránsito por el proceso de externación manicomial. Esto ocurre a nivel de la escena cotidiana de tomar a cargo las tareas propias de la esfera doméstica. En base a estas situaciones que testimonian la autonomía en la escala cotidiana, molecular, realizamos un análisis que permitió utilizar la serie autonomía-autogestión-micropolítica.

\section{BREVE CONCLUSIÓN}

A modo de cierre, nos convoca poner de relieve los aportes sustanciales y distintivos que supone el uso de esta metodología. Por un lado, señalamos la multi-referencialidad para generar evidencia significativa; en segundo término, la democratización de voces expresadas de distintos modos y la posibilidad de que el conocimiento generado se coloque en un plano de sentido social-político en referencia al acceso a derechos. La identificación empírica de estos rasgos nos permite dilucidar que esta metodología no es estrictamente investigación y acción sino es una concepción del conocimiento ligada a la vida como posibilidad de transformación, de disfrute estético y ético, de proposición de exploración sobre lo que aún no se conoce, es decir, lo inesperado.

Es menester señalar que la posición de co-investigación admite su inscripción para el caso de la salud mental en base a referencias a campos disciplinares diversos, tal como dejamos señalado en este trabajo. Los aportes de pensadores que se han ocupado de avanzar en la pregunta por el lugar que es otorgado al otro en las prácticas de salud y de manera más precisa, el foco en la circulación del poder y el saber para la generación de conocimientos, permiten dar sustento a la mirada aquí presentada a modo de conclusión. Entre ellos, en el campo de la filosofía política, Foucault (1977) conceptualiza los efectos de poder que se encuentran entrelazados al saber. En un dispositivo, estos efectos se distribuyen de manera irregular permitiendo hacer visibles las asimetrías en tanto figuras aptas para identificar las desigualdades que subyacen en las prácticas. Para pensar una lógica democratizante que capilarice las acciones tanto de investigación como de salud, nos apoyamos además en algunas nociones clave que Castoriadis (1993) nos ha legado. La noción de democracia comprendida como un régimen de sentido que excede lo meramente procedimental nos permite leer las realidades de estudio que vamos construyendo en colaboración con otros. Asimismo, este autor acuña la idea de proyecto de autonomía, que ha brindado un apoyo conceptual a la hora de desarrollar un saber-hacer en situación que busca la participación igualitaria en cada instancia de poder y saber que incluye una toma de decisión. La potencia de la autonomía para leer las prácticas de sa- 
lud mental se refleja en la puesta en marcha de la acción democratizante desde la participación.

Historizar el avance democratizante en salud mental, comenzando como presentamos aquí por hacer visibles diferentes marcos conceptuales, prácticas disciplinares y escrituras en distintos idiomas y geografías, nos permite terminar con evocación a los movimientos europeos de Antipsiquiatría, Psiquiatría Democrática, y Psicoterapia institucional, ya que fueron estos los referentes para la emergencia del proceso de desmanicomialización que continúa en desarrollo en Latinoamérica. Todos ellos perpetraron la lucha colectiva en orden a la democratización de los poderes y saberes que constituyen las acciones de salud.

\section{REFERENCIAS}

ACOSTA, C. (2019). “Aportes al estudio de la autonomía como proyecto: análisis de aprendizajes vinculados a la práctica de la autogestión y sus relaciones con la construcción de la salud mental como derecho" (Tesis doctoral) Universidad Nacional de Córdoba, Córdoba, Argentina.

BEJARANO, F., BURIN, D., DAGA, A., HERAS, A., PACHECO, M., SOSA, G, GARCÍA, E., RUSCITTI, I. GONZÁLEZ G. ONETO, G., SCHMUK, M. y YUJNOVSKY, N. (2019). "La Red de cooperativas sociales. La potencia del rizoma". En $\mathrm{Ha}$ ceres colectivos. Investigaciones y Experiencias en Salud Mental y Adicciones en Argentina. Buenos Aires, Argentina: Ministerio de Salud y Desarrollo Social de la Nación, pp. 157-168.

BAREMBLITT, G. (1982): "Notas estratégicas a respeito da orientaçao da dinamica de grupos na América Latina." Em Gregorio Baremblitt (coord.), Grupos. Teoria e Técnica (pp. 11-37). Rio de Janeiro, Brasil: IBRAPSI.

BARGAL, D. (2006): "Personal and intellectual influences leading to Lewin's paradigm of action research: Towards the 60th anniversary of Lewin's 'Action research and minority problems' (1946)." Action Research, 4(4), 367-388. https://doi. org/10.1177/1476750306070101

CASTORIADIS, C. (1993): "La cuestión de la democracia. Posibilidades de una sociedad autónoma." Revista Zona Erógena, 44. Buenos Aires, Argentina.

CASTORIADIS, C. (2005): Los dominios del hombre. Las encrucijadas del laberinto. Barcelona: Gedisa.

DELEUZE, G., \& GUATTARI, F. (1988): Mil Mesetas. Capitalismo y Esquizofrenia. Valencia: Pre-textos.

DUSSEL, E. (1973). Para una ética de la liberación latinoamericana, Tomo I. Buenos Aires, Argentina: Siglo XXI Editores.

FABRIS, F. A. (2007): Pichon-Rivière, un viajero de mil mundos: génesis e irrupción de un pensamiento nuevo. Buenos Aires, Argentina: Polemos.

FABRIS, F. (2009): "Pichon-Rivière, irrupción y génesis de un pensamiento." Revista Intersubjetivo de Psicoterapia Psicoanalítica y Salud, 1(10), 11-28.

FABRIS, F. (comp.) (2014): Pichon-Rivière como autor latinoamericano. Buenos Aires, Argentina: Lugar Editorial.

FALS BORDA, O. (1999). Orígenes universales y retos actuales de la IAP. Análisis Político, (38), 73-90. Recuperado a partir de https://revistas.unal.edu.co/index.php/ anpol/article/view/79283 
FALZON, M. (ed.). Multi-Sited Ethnography: Theory, Praxis and Locality in Contemporary Research. Farnham and Burlington: Ashgate.

FOUCAULT, M. (1977). Historia de la sexualidad. Buenos Aires: Editorial siglo XXI.

FREIRE, P. (1969). La educación como práctica de la libertad. Montevideo, Uruguay: Tierra Nueva.

GENAT, B. (2009): Building emergent situated knowledges in participatory action research. Action Research, 7(1), 101-115. https://doi.org/10.1177/1476750308099600

GRINBERG, L. LANGER, M. \& RODRIGUÉ, E. (1961 [1957]): Psicoterapia del grupo. Buenos Aires, Argentina: Paidós.

GUATTARI, F. \& ROLNIK, S. (2005). Micropolítica. Cartografías del deseo. Buenos Aires: Tinta Limón Ediciones.

HERAS, A.I. (2014). Lógica colaborativa y generación de conocimiento colectivo. Alcances y tensiones en la relaciones investigación-sociedad. Población \& Sociedad, 21(2), 137-150.

HERAS, A. I. (1999). Taking Action with Family and Community Members: Critical Pedagogy as a Framework for Educational Change. Advances in Confluent Education, 2, 73-107. Zulmara Cline y Juan Necochea editores, JAI Press Inc. Stamford, Connecticut: U.S.A.

HERAS, A. I. (1993). The construction of understanding in a sixth grade bilingual classroom. Linguistics and Education, 5(3\&4), 275-279.

HERAS, A. I. (1995). Living Bilingual, Interacting in Two Languages: An Ethnographic and Sociolinguistic Study of a Fourth Grade Bilingual Classroom (Tesis doctoral). Santa Barbara: Universidad de California.

HYMES, D. (1974): Foundations in Sociolinguistics: An Ethnographic Approach. Philadelphia: University of Pennsylvania Press.

KUSCH, R. (2000). Obras completas. Volumen I. Rosario, Argentina: Fundación Ross.

LEWIN, K. (1946/1948): "Action research and minorityproblems." In G. W. Lewin (Ed.), Resolving social conflicts (pp. 201-216). New York: Harper\&Row.

LEWIN, K. (1947a): Frontiers in group dynamics, I. Human Relations, 1(1), 2-38.

LEWIN, K. (1947b): Frontiers in group dynamics, II. Human Relations, 1(1), 143-153. MARTÍN BARÓ, I. (1986). Hacia una psicología de la liberación. UCA.

PICHON-RIVIÈRE, E. (1995). Diccionario de términos y conceptos de psicología y psicología social. Nueva Visión.

PICHON-RIVIÈRE, E. (2008, 26 reimpresión). Teoría del vínculo. Nueva Visión.

PICHON-RIVIĖRE, E. (1969a). Estructura de una escuela destinada a la formación de psicólogos sociales. En El proceso grupal. Del psicoanálisis a la psicología social. Nueva Visión, pp. 149-160.

PICHON-RIVIĖRE, E. (1969b).Una teoría del abordaje de la prevención en el ámbito del grupo familiar. En El proceso grupal. Del psicoanálisis a la psicología social. Nueva Visión, pp. 185-190.

PICHON-RIVIĖRE, E. (1971). El proceso grupal. Del psicoanálisis a la psicología social. Buenos Aires: Ediciones Nueva Visión.

POZZO, M.I, MIANO, A. y HERAS, A.I. (2018) La etnografía colaborativa en la formación en investigación en Ciencias de la Educación. Pensamiento Actual,18, p. 102 $-113$

QUIROGA, A. (1986): “Enrique Pichón Rivière.” En El proceso educativo según P. Freire y E.Pichón Riviére, pp. 13-31. Edigraf: Buenos Aires, Argentina 
ROCKWELL, E. (2005): "Del campo al texto. Reflexiones sobre el trabajo etnográfico." Conferencia presentada en Sesión Plenaria Primer Congreso de Etnología y Educación, Universidad Castilla-La Mancha.

RODRIGUÉ, E. (2000): El libro de las separaciones. Sudamericana: Buenos Aires, Argentina.

ROZITCHNER, L. (2012 [original de 1972]): Freud y los límites del individualismo burgués. Buenos Aires, Argentina: Ediciones de la Biblioteca Nacional.

SAIDÓN, O. (2014): "Políticas de salud mental." En Voces en el Fénix, 5(41), 48-57.

SAIDÓN, O. (2012): "La clínica de Guattari y los post-guattarianos." En Berti, Gabriela (coordinación editorial). Félix Guattari, los ecos del pensar. Entre filosofía, arte y clínica. Haka Books: Barcelona, España.

SAIDÓN, O. (2015): Ahora sí. Reflexiones sobre la implicación. En Revista Psicoanalítica, Número especial sobre Instituciones. Editorial CPN (Centro Psicoanalítico del Norte). Buenos Aires, Argentina.

SIRVENT, M.T. (1990). Investigación participativa aplicada a la renovación curricular. Capítulo 1. Buenos Aires: Instituto de Ciencias de la Educación, FFyL, UBA.

TASSIN, E. (2012). De la subjetivación política. Althusser/Rancière/ Foucault/Arendt/ Deleuze. Revista de Estudios Sociales (43), p p. 36-49 Universidad de Los Andes Bogotá, Colombia. 
